\title{
Shift of effective lightning areas during pre to post period of solar cycle minimum of 2008-2009 as determined from Schumann resonance studies at Agra, India
}

\author{
Birbal Singh ${ }^{\star}$, Devbrat Pundhir
}

RBS Engineering Technical Campus, Department of Electronics and Communication Engineering, Bichpuri, Agra, India

\author{
Article history \\ Received May 30, 2014; accepted December 26, 2014. \\ Subject classification: \\ Schumann resonance, Thunderstorm activity, Effective lightning areas.
}

\begin{abstract}
Employing a set of 3-component search coil magnetometer, Schumann resonance studies have been in progress at Agra (Geograph. lat. $27.2^{\circ} \mathrm{N}$, long. $78^{\circ} \mathrm{E}$ ), India since 01 April, 2007. We have analysed the data for two periods; first from 01 April, 2007 to 31 March, 2008 (period-I), and then from 01 March, 2011 to 29 February, 2012 (period-II) which correspond to pre and post periods of solar cycle minimum of 2008-2009. From the diurnal variation of first mode intensity and frequency, we study the seasonal variations of global thunderstorm activity, effective source distance and level of lightning during both the periods. We show that world thunderstorm activity shifts to summer in the northern hemisphere as the effective source distance approaches close to the observer, and the level of intense lightning shifts from the month of July, 2007 in period-I to August, 2011 in period-II. This is supported by Lightning Imaging Sensor (LIS) satellite data also. A possible explanation in terms of increasing solar activity is suggested.
\end{abstract}

\section{Introduction}

The phenomenon of Schumann resonance (SR) occurring in the earth-ionosphere cavity as a result of resonance between direct and round the globe propagation of extremely low frequency (ELF) waves radiated from lightning discharges was first predicted by Schumann [1952] and experimentally verified by Balser and Wagner [1960]. The SR appears in the form of standing waves at the frequencies of $8,14,20,26 \ldots \mathrm{Hz}$ which are known as SR modes. Owing to its widespread applications in the studies of global thunderstorm activities, ground surface temperature, lower region of the ionosphere, and forecast of monsoon etc. extensive research work has been done in this field and a detailed description of the early work is presented in an excellent monopgraph by Nickolaenko and Hayakawa [2002]. Some very interesting re- cent results which have come out of the extensive morphological and varying geophysical condition studies have been presented by many workers. For example, Price and Melnikov [2004] have studied the diurnal, seasonal, and inter-annual variations in the SR phenomenon and have found three dominant maxima in the diurnal cycle related to lightning activity in south-east Asia, Africa, and South America. Further, the largest global lightning activity occurs during the northern hemisphere summer (JJA), with the southern hemisphere summer (DJF) having the least lightning around the globe. Greenberg and Price [2007] have compared the ELF data on the ground with the Optical Transient Detector (OTD) data on satellite and found discrepancies in lightning activities between the Asian and South American centers. To understand the relative strength of the different source regions they calculated the attenuation rate for the electromagnetic waves in SR band in both the NorthSouth and East-West direction reaching their station. They found that the simulated magnetic field relative amplitude based on OTD observations showed a peak at 2000 UT corresponding to American source 40\% larger than African and Asian sources, whereas in the East-West direction the African peak at 1400 UT was 80\% larger than the American peak and Asian peak was not visible. Hayakawa et al. [2005] and Nickolaenko et al. [2006] have reported anomalies in SR bands as they found increase in the intensity of fourth harmonic due to earthquake which were interpreted in terms of superposition of direct ELF signals from a distant thunderstorm source in America with those scattered from a conducting disturbance in atmosphere over the epicenter of earthquake. Williams and Satori [2007] have studied the solar radia- 
tion-induced changes in ionospheric height and SR waveguide on different time scales. They find that, in general, order of magnitude changes in radiation are needed to cause relative changes in ionospheric height as large as $10 \%$ as in the case on both the diurnal and 11-year time scales. On day-night asymmetry of the earth-ionosphere cavity and solar terminator effects Nickolaenko and Hayakawa [2002] concluded theoretically that the amplitude variations to be expected at the sunrise/sunset termination line are practically undetectable. Figure 7 of Nickolaenko et al [2006] demonstrates that the lens-like "terminator" pattern is pertinent to the global thunderstorm activity itself. This is why a uniform earth-ionosphere cavity provides the "terminator effect" similar to observations (in the UT) at an orbitary observatory. The confusion with the ionosphere local height occurred due to closeness of the start-stop times of the planetary lightning activity with the sunrise and sunset moments at the middle East and central Europe. Similar inference was drawn also by Penchony et al. [2007] who observed that the SR field variations are governed primarily by the variations in source intensity and source-receiver geometry and that the effect of the day-night asymmetry in the ionosphere is secondary. In contrast to the above, Melnikov et al. [2004] presented observational evidence for the sunrise/sunset terminator effect based on multistation SR observations with hourly time resolution and found steep increase/decrease of SR amplitude during terminator times. A similar result was found also by Ondraskova et al. [2007] at Modra observatory, Slovakia for the first four modes of electric field component. The later results were supported also by the observations of Satori et al. [2007a] who found jump-like increase of SR amplitude between the local ionosphere and surface sunrise times and sharp decreases between the local surface and ionospheric sunset times. The SR phenomenon is due to contribution from both the global and local thunderstorm activities. In order to separate the local contribution from the global one Sentman and Fraser [1991] have suggested a technique which has been applied by many workers [Pechony and Price 2006, Nickolaenko and Hayakawa 2008, Nickolaenko et al 2011]. It is shown that local modulation function depends primarily on source-receiver distance geometry and it can be separated with the technique suggested by Sentman and Fraser [1991]. Ondraskova et al. $[2009,2011]$ have studied the decrease of SR frequencies and changes in effective lightning areas toward the solar cycle minimum of 2008-2009 using the data from Modra observatory Slovakia. The extraordinary fall in the first mode frequency was interpreted in the light of the suggestion made by Satori et al [2005] that the reduction in $\mathrm{X}$-ray radiation causing decrease in the ionospheric conductivity may be attributed responsible for the observed effect. They further calculated the effective thunderstorm areas from the monthly mean diurnal frequency range (DFR) of the electric field component. They found that the difference in the northern and southern hemisphere summer areas not only declined with the decreases in solar activity but almost vanished during the deep solar minimum of 2008-2009. Semi-annual variation in the areas dominates in the years of the deep solar minimum. In our recent paper [Tyagi et al. 2013, hereafter paper I] we have studied the characteristics of global thunderstorm activities extracted from SR data for the period of 01 April, 2007-31 March, 2008. In the present paper we extend some of the studies made earlier in paper I especially those related to variation of global thunderstorm activities and compare the results. Then, we consider the first mode frequency and intensity of the magnetic field data to study the effective source distance and level of lightning for both the periods 01 April, 2007-31 March, 2008 (period-I) and 01 March, 2011-29 February, 2012 (period-II) which are pre and post periods of solar cycle minimum of 2008-2009 and compare the results.

\section{Experimental set up and method of data processing}

The details of experimental set up and method of data processing are not available in paper I. However, these details are available in our another paper [Singh et al. 2014]. Here, we mention the details briefly as follows; We are using a 3-component search coil magnetometer (LEMI-30) which has been procured from Lviv center of Institute of Space Research, Ukraine. The search coils are buried under-ground in a relatively noise free area in Agriculture fields of the Bichpuri campus of R.B.S. college, Agra which is located $12 \mathrm{Km}$ west of Agra city in rural area. The three sensors are oriented in geographical North-South (X-component), East-West (Y-component), and vertical (Z-component) directions and each of them has following specifications;

Dimension: $\quad 870 \mathrm{~mm}$ (length), $85 \mathrm{~mm}$ (diameter) $\begin{array}{lc}\text { Frequency range: } & 0.001-30 \mathrm{~Hz} \\ \text { Measuring range: } & \pm 200 \mathrm{nT}\end{array}$

Transformation factor $0.001-1 \mathrm{~Hz}$ : 1-30 Hz: $20 \times \mathrm{f} \mathrm{mV} / \mathrm{nT}$ $20 \mathrm{mV} / \mathrm{nT}$

Auxiliary output gain: $20 \mathrm{~dB}$

Magnetic noise level at $0.01-10 \mathrm{~Hz}$ : $\leq 20 \mathrm{pT} / \mathrm{Hz}^{1 / 2}-\leq 0.04 \mathrm{pT} / \mathrm{Hz}^{1 / 2}$

Mains interference rejection:

$>60 \mathrm{~dB}$

The LEMI-30 system has sampling rate of $256 \mathrm{~Hz}$ and sends these samples to dedicated PC. The LEMI-30i soft- 
ware in the PC takes average of each four samples simply by summing and dividing so that a binary file containing data at the sampling rate of $64 \mathrm{~Hz}$ is stored in the PC. The recorded data on PC in amplitude-time may be seen in frequency-time (dynamic spectra) by performing spectral analysis using FFT available in MATLAB with 1024 words of data length (temporal resolution $=16 \mathrm{sec}$, frequency resolution $=0.06 \mathrm{~Hz}$ ) at a time. The power spectral density (PSD) of the input signal is evaluated using Welch spectral technique [Welch 1967] which uses averaged modified periodograms. The PSD are prepared for each one hour data (230400 data points) and a Hamming window of 1024 data points with sliding of half the window is used to compute the modified periodogram of each segment. In this method the spectrum obtained per hour is the average of 450 spectra with 1024 data points. The Welch method is closely related to the method of complex demodulation described by Bingham et al [1967] and hence a separate method to extract SR modal frequencies in the analysis of data is not applied. An example of dynamic spectrum and corresponding PSD obtained from the data recorded by the $\mathrm{X}$-component of the sensors on 18 February, 2012 is presented in Figure 1a and $1 \mathrm{~b}$ respectively. The PSD shows amplitude in $\mathrm{dB}$ which is converted in intensity (in $\mathrm{nT}^{2} / \mathrm{Hz}$ ) and the results corresponding to Figure $1 \mathrm{~b}$ is shown in Figure 1c. In Figure 1a the time is shown in UT which is related with local time $\mathrm{LT}=\mathrm{UT}+5.5 \mathrm{hrs}$. Here, it may be noted that Figure 1a resembles in many respect with Figure 1a of paper I especially the distribution of spikes in the coloured figure. However, these two figures are different because they were observed on different days, and the differences are indicated by PSDs also. Since the time of measurement is same during which a passenger train passes every day, similar spikes appear in both the figures. The detailed examination of the quality of the SR data shows that due to local noises including the movement of trains on a railway track (about 300 $\mathrm{m}$ away in East-West direction) the X-component is affected least, the Y-component moderately, but the Zcomponent severely. Hence, we use X-component data mostly in our analysis. However, we use Y-component data also wherever necessary by applying careful selection of the data. In order to further process the data we write a program in MATLAB to extract hourly information about the peak frequencies and intensities (determined by the program itself) of three modes and arrange them in separate columns in EXCEL. In this way, we prepare a time series for the frequencies and intensities of the SR data for 24 hours each day and then for 12 months from 01 March, 2011 to 29 February, 2012. This time series is used to deduce various characteristics of SR phenomenon observed at Agra station.
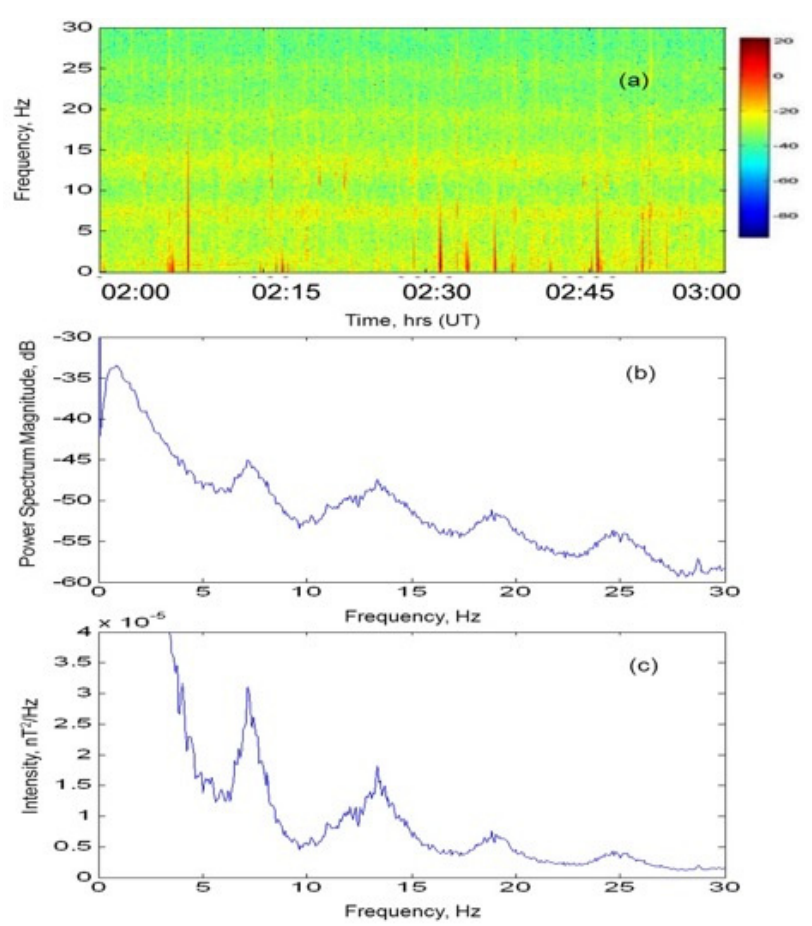

Figure 1. (a) Frequency-time spectrogram of the ULF/ELF data recorded on 18 February, 2012 at Agra showing four Schumann resonance lines (b) Power spectrum density (PSD) of the data in Figure $1 \mathrm{a}$ and (c) corresponding intensity-frequency variation.

\section{Lightning Imaging Sensor (LIS) data}

While electromagnetic radiations emitted by lightning form an important tool for the study of global lightning, another very useful advanced space based tool is the Optical Transient Detector (OTD) which allows us to understand the spatial and temporal distribution of lightning around the globe, Christian et al. [2003] have given a detailed description of instruments and their characteristics and measurements used to construct lightning climatology maps demonstrating the geographical and seasonal distribution of lightning activity for the globe. However, since OTD satellite stopped working prior to the beginning of measurements at Agra, we have used data for this paper from Lightning Image Sensor (LIS) available on the website: http: / / thunder.nsstc.nasa.gov/ data / query/distribution/html collected on Tropical Rainfall Measurement Mission satellite (TRMM) with the following coverage;

Location

Sub-Tropical

Latitude range $\pm 40^{\circ}$

Longitude range $\pm 180^{\circ}$

Spatial resolution 3-6 Km

Temporal resolution :

ORBIT

The data have been used for both the periods of 01 April, 2007 to March 31, 2008 and 01 March, 2011 to 29 February, 2012. It may be noted here that both the electromagnetic (SR) and OTD/LIS measurements suffer 
due to some drawbacks. The problem with SR measurements lie in a too high global rate of strokes of about 100 events per second. With the basic $8 \mathrm{~Hz}$ frequency of SR we have about 12 independent pulses arriving at an observer during the single period of this frequency. It is obvious that signals will overlap severely making impossible their separate processing. The main problem in OTD observations is that an observer does not know what is going on outside the frame of optical device at any particular time. Furthermore, there are 'invisible' strokes, i.e. the discharges whose optical emissions do not reach the top of the cloud [Nickolaenko and Hayakawa 2002]. In contrast to the above, a space sensor capable of mapping both inter cloud and cloud-tocloud lightning discharges from geostationary orbit during day and night with a spatial resolution of $10 \mathrm{Km}$ and a detection efficiency of $90 \%$ has been developed. In addition, this sensor, which is called the Lightning Mapping Sensor (LMS) can monitor storms on a continual basis. The combination of modern solid state mosaic focal planes with extensive on-board signal processing in the LMS provides a powerful technique for the detection of weak background-contaminated signals and permits the detection of lightning during the day [Christian et al. 1989]. Such data could be very helpful in defining the source diameter of prominent lightning regions. However, the capabilities of lightning detection from geostationary orbit are limited by several factors which include visible area limited to a nominal field of view, resolution depends on geographical position and drops with distance from nadir, earth observation is interrupted and detection efficiency reduces if a spinning satellite is used, light scattering in clouds limits the location to $8-10 \mathrm{Km}$ etc. [Finke and Hauf 2002]. Hence, we have employed the LIS data only in this paper. As far as SR observations are concerned we have mentioned in section 2 how movements of trains on nearby tracks produce spikes in our data whose intensities are not as significant as to appear in the PSDs of the SR data. Especially, the use of North-South (X-component) component of the data permits us to process the SR characteristics without much problem. In general, the X-components contain more power than Y-components due to the fact that energies generated by lightning from nearby thunderstorm center (Asian source) and those from American source transmitted along East-West direction are higher than those transmitted in the North-South direction. The raw temporal data show sporadic spikes in amplitude due to passage of trains but no saturation effect is observed. The noises arising from other sources (electrical and atmospheric noises) are sometimes serious and do affect the quality of data. However, they are occasional and due precaution is taken while processing the data.

\section{Observational results}

4.1 Monthly distribution of thunderstorm activities

In paper I, we have made a correlative study between the first mode SR intensity observed at Agra and Lightning Imaging Sensor (LIS) data for the period 01 April, 2007-31 March, 2008 (period I). Here, we make a similar study for the period 01 March, 2011-29 February, 2012 (period II). Both the results are shown in Figure2a,b. The dark histograms show the LIS data whereas the light dark histograms show the SR intensity. The intensity variations shown in both parts of the figure are integrated intensity of the first three modes because a modal SR intensity depends not only on the activities of the source but also on source-observer geometries. From a general scanning of the data we find that the intensity of the second and third modes constitutes approximately 40 percent of the total intensity of the three modes. The consideration of integrated intensity may effectively neutralize the geometrical factors. It may be noted from Figure 2 that peak thunderstorm activity occurred in the month of July during period I whereas the same occurred in the month of august during period II.The correlation coefficients between the SR intensity and LIS data for the two periods are 0.81 and 0.70 respectively. In section 3, we have indicated the short falls in the measurements of both the SR and OTD data which may be responsible for the low correlation coefficient. The correlation coefficients are low but not poor possibly because both the SR and LIS data are influenced more by south-Asian thunderstorm center (which is just $3000 \mathrm{Km}$ from the observing stations) in spite of the fact that both the measurements respond to global phenomena. Further, looking at the two panels of Figure 2 we find that the LIS data in the upper panel corresponding to period I are 3-5 times larger than those in the lower panel corresponding to period II, whereas the magnitude of SR intensities during the two periods are opposite to each other i.e. the intensities are 2-3 times larger in period II than these in period I. These anomalies in SR intensity and LIS data will be discussed later.

\subsection{Effective source distance and level of lightening}

The dependence of frequency range, i.e. the maximum value minus its minimum value $\left(\mathrm{dF}_{1}=\mathrm{f}_{1}\right.$ max $\mathrm{f}_{1}{ }^{\mathrm{min}}$ ) has been used for establishing the effective size of zone occupied by the world wide thunderstorm activity from the SR records [Nickolaenko and Rabinovicz 1995, Nickolaenko et al. 1998]. The diurnal variation in $\mathrm{dF} 1$ is related to movement of the lightening region along the equator from one continent to another around the globe. The physical background is based on the discontinuity of $\mathrm{f1}$ when the point source crosses the nodal 

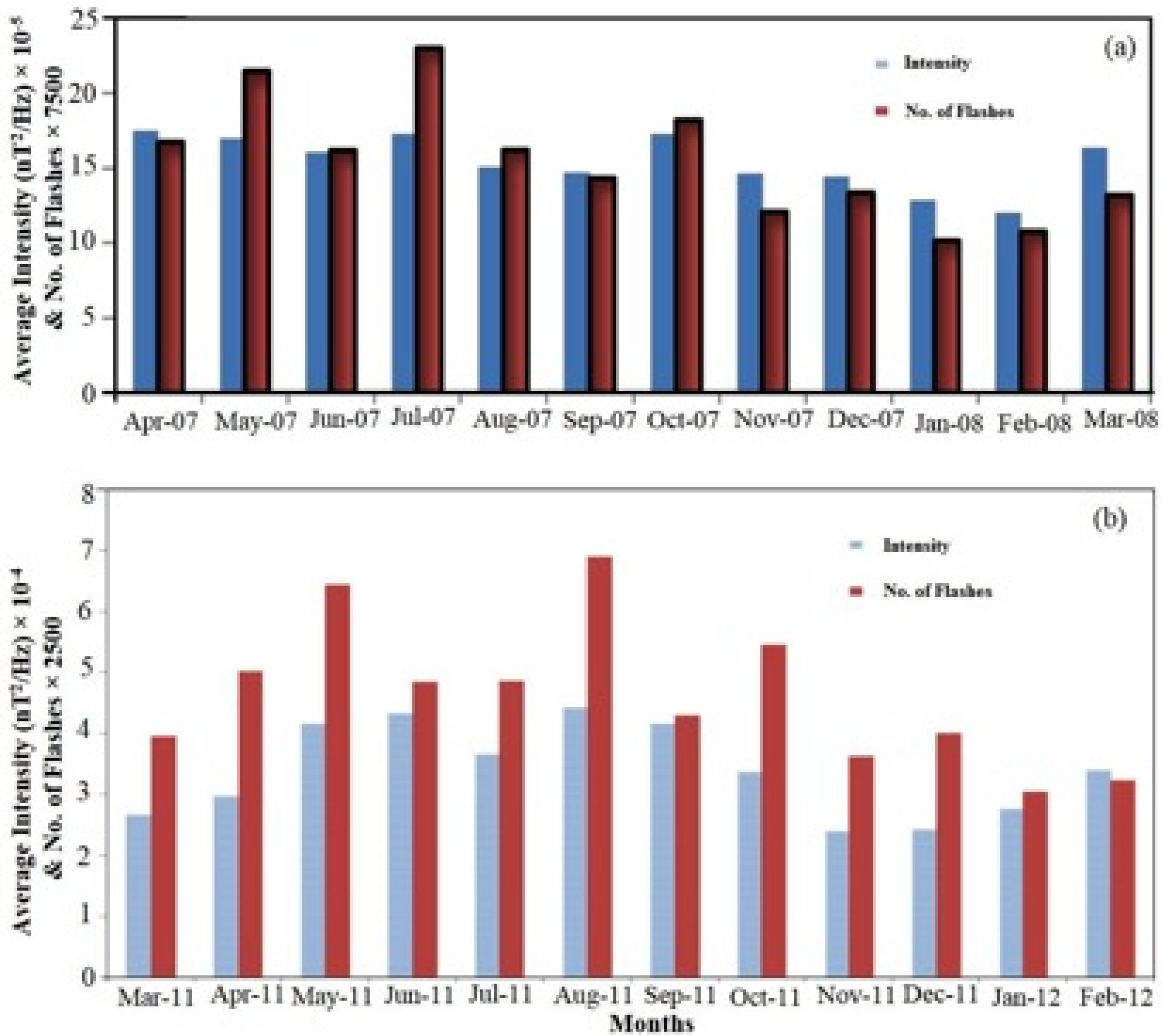

Figure 2. (a) Correlation between the global distribution of thunderstorm activities deduced from SR data at Agra for the period of 01 April, 2007 - 31 March, 2008 with Lightning Image Sensor (LIS) data on the satellite (b) the same as above but for the period of 01 March, 2011 - 29 February, 2012.

line. When the source becomes wider the discontinuity turns into the abrupt transition from small to great frequency values. The variation in $\mathrm{df}_{1}$ vanish when the lightening sources uniformly cover the globe. A detailed description of these results are given in section 4.1.5 and 4.2.2 of Nickolaenko and Hayakawa [2002]. Here, it may be emphasized that $\mathrm{dF}_{1}$ is connetcted with the source size only when measured in the $\mathrm{E}_{z}$ field component. It reflects the daily alternations of the source distance when measured in the horizontal magnetic field. However, the above results are based on measurement of vertical electric field component and one has to measure the peak frequency in the $<|\mathrm{E}(\mathrm{f})|^{2}>$ spectra to evaluate the source width [Nickolaenko and Hayakawa 2002, Satori et al. 2009, Ondraskova et al. 2011]. The reason for why electric field measurement is more important than magnetic field measurement for the above purpose may be understood from Figure 3 in which power spectra for both $\mathrm{E}$ and $\mathrm{H}$ fields in the vicinity of the first mode over the distance-frequency plane is presented (the point source is considered). This figure has been received with the courtesy of Prof. A.P. Nickolaenko. Although the figure is in black and white the results are clearly evident. The left frame shows the power spectra of the vertical electrical field $\mathrm{E}_{\mathrm{z}}$, and the right frame shows the dynamic spectrum of the horizontal magnetic field $\mathrm{H}_{\phi}$. The obvious distinction of these spectra is the node around the $10 \mathrm{Mm}$ distance in the $\mathrm{E}_{\mathrm{z}}$-field. The horizontal magnetic field component has the "tilted" maximum here. The tilt is conditioned by the influence of higher resonance modes, and the same influence makes the nodal line asymmetric. As a result, the $f_{1}$ frequency in the $E_{z}$ power spectrum has the discontinuity at $\mathrm{d}=10 \mathrm{Mm}$. Since we do not have electric filed measurement setup at Agra station we do not use $\mathrm{dF}_{1}$ to evaluate source width during 


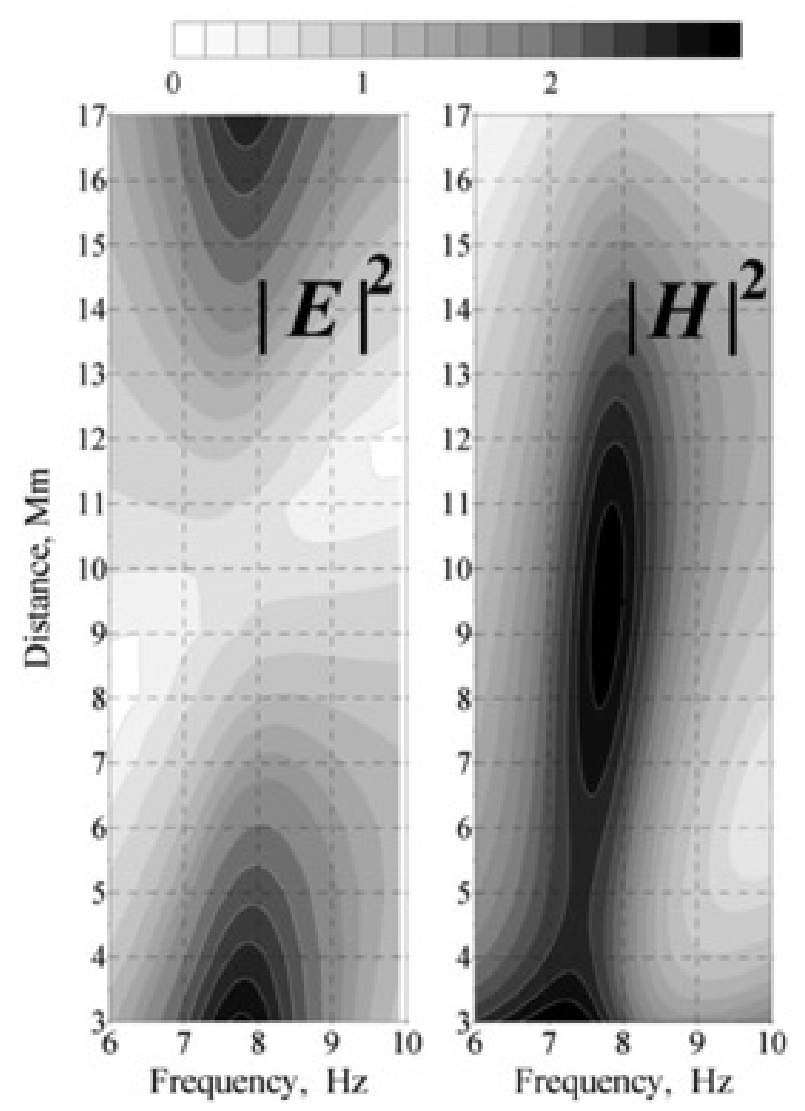

Figure 3. Power spectra of the electric filed $\mathrm{E}$ (left) and magnetic field $\mathrm{H}$ (right) in the vicinity of the first mode SR over the distance-frequency plane (received with the courtesy of Prof. A.P. Nickolaenko)..

the two periods under consideration using magnetic field data. However, some of the parameters of SR magnetic field data such as $f_{1}$ and intensity $I_{1}$ can be used to derive effective source distance (from $f_{1}$ ) and, after words, the effective level of lightning activity (from $\mathrm{I}_{1}$, and effective source distance $\mathrm{D}$ ). For this purpose we follow the equation [Nickolaenko and Hayakawa 2002],

$f_{1}(d)=a_{0}+a_{1} d+a_{2} d^{2}+a_{3} d^{3}+$

Here $\mathrm{d}$ is the source-observer distance in $\mathrm{Mm}, \mathrm{a}_{\mathrm{o}}=$ $6.71336, \mathrm{a}_{1}=0.181732, \mathrm{a}_{2}=-0.008612582, \mathrm{a}_{3}=-0.0001477123$. This equation can be used to evaluate peak frequency from the known source distance. If we rotate the coordinate axes of the graph obtained from above and plot the same data in the form $d\left(f_{1}\right)$ the polynomial fit provides [see Nickolaenko 2014]

$\mathrm{d}\left(\mathrm{f}_{1}\right)=\mathrm{b}_{\mathrm{o}}+\mathrm{b}_{1} \mathrm{f}_{1}+\mathrm{b}_{2} \mathrm{f}_{1}^{2}+\mathrm{b}_{3} \mathrm{f}_{1}{ }^{3}+\mathrm{b}_{4} \mathrm{f}_{1}^{4}$

where $b_{0}=207049, b_{1}=-110796, b_{2}=22229.7, b_{3}=-1982.03$, $\mathrm{b}_{4}=66.2696$. We can use this equation to evaluate the source distance from the experimental peak frequency. Since the real source width is non-zero, this distance should be regarded as effective one. The results of these calculations are presented in graphical forms for the

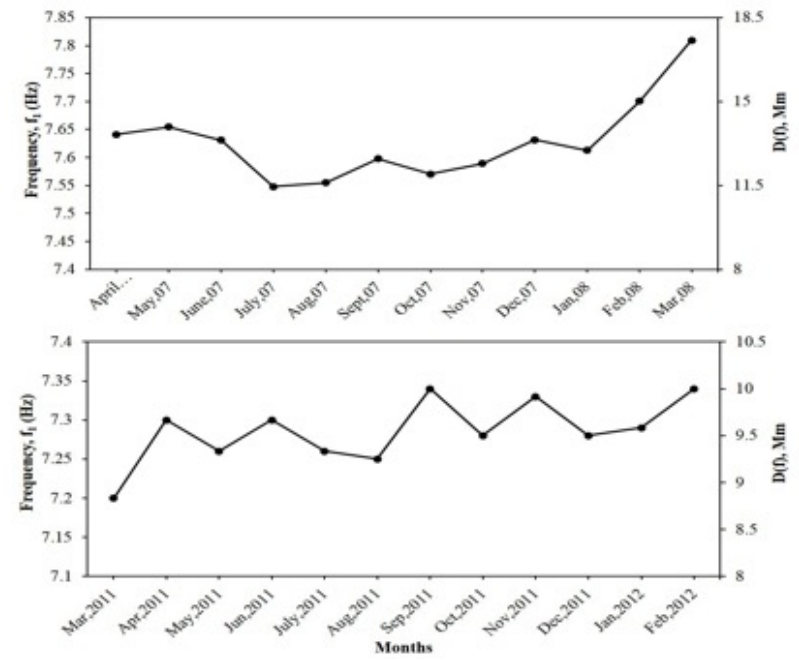

Figure 4(a). Seasonal variation of first mode SR frequency of the North-South component (X-component) of the horizontal magnetic field for period-I (upper panel) and period-II (lower panel). The ordinate on the right shows the corresponding source-observer distance.

two periods in Figure. 4a to 4c. In the two panels of Figure $4 \mathrm{a}$ are shown the seasonal variation of first mode SR frequency of the North-South component (X-component) of the horizontal magnetic field and corresponding source-observer distance derived from equation (2) for period-I (upper panel) and period-II (lower panel) respectively. The range of frequencies shown on the ordinate of the panel of period-I is higher than that on the ordinate of panel-II. An explanations for the decrease in the first mode frequency in periodII and increasing trend with the months will be given in discussion section. Here, we see that in both the periods the summer thunderstorms shift towards the northern hemisphere (NH) summer (May-August) as the minimum source-observer distances reach around 12.4 $\mathrm{Mm}$ and $9 \mathrm{Mm}$ respectively. Annual drifts of these sources in the two periods are approximately $4 \mathrm{Mm}$ and
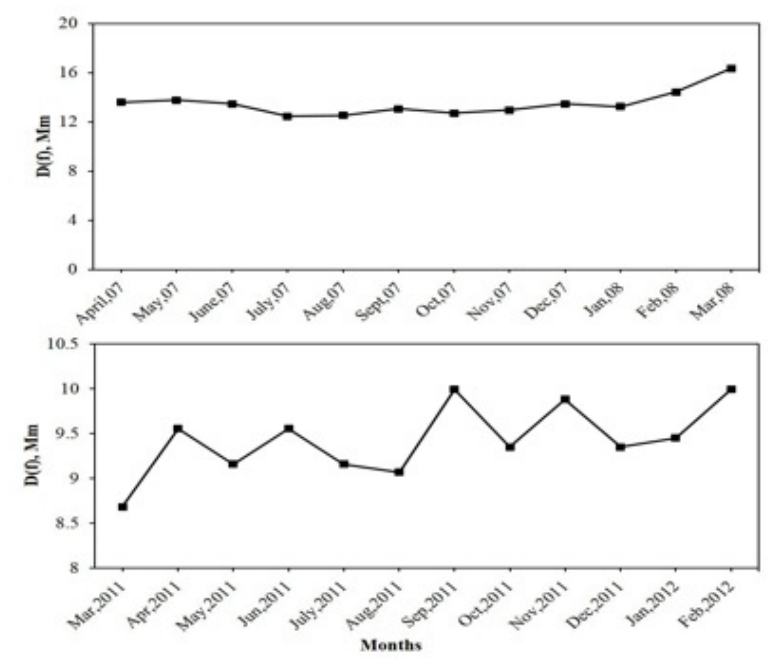

Figure 4(b). Seasonal variation of source-observer distance derived from observed first mode frequency for the two periods. 

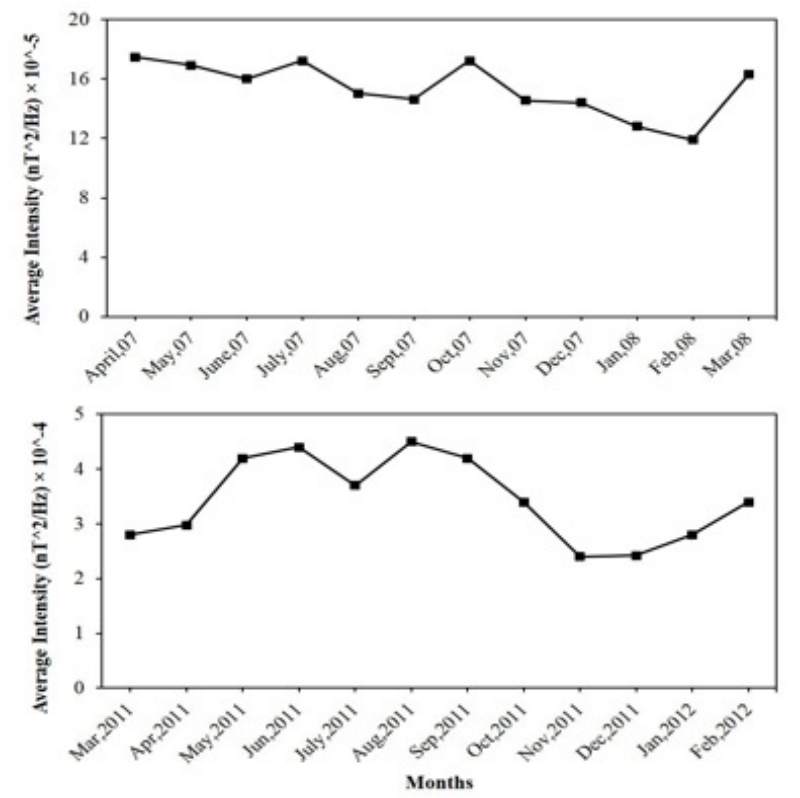

Figure 4(c). Seasonal variation of first mode intensity for the two periods.

$1.3 \mathrm{Mm}$ corresponding to $36^{\circ}$ and $12^{\circ}$ respectively. The seasonal variations of the effective source-observer distance derived from the first mode SR frequency for the two periods are separately presented in Figure $4 \mathrm{~b}$. It should be noted that the obtained estimates of the distances to the source in the two periods are more than $10 \mathrm{Mm}$ (for period-I) and less than $10 \mathrm{Mm}$ (for periodII) respectively. Clearly visible are the seasonal drift of world thunderstorms to the NH summer. Satori [2003] has carried out long-term monitoring of the $\mathrm{E}_{z}$ component of the SR parameters (peak amplitude and peak frequencies) and provides very useful information on seasonal motion of the global lightning activity [see also Hayakawa et al 2010]. The variations of average intensity of the first mode SR corresponding to North-South components for the two periods are shown in the upper and lower panels of Figure 4c. The intensity data are the same as shown in Figure 2. However, they are shown here to be examined in the light of the variation of source-observer distances presented in Figure $4 \mathrm{~b}$. Here, we see that the intensities are higher in the summer months (May-August) when the source-observer distances are lowest indicating that nearer the sources larger are the intensities. An interesting point to be noted here is that the level of maximum intensity corresponding to intense lightning exists in the months of July in period-I whereas the same exists in the months of August in period-II. These months of largest SR intensities are supported by LIS data also (see Figure 2). Hence, we can say that there is a shift of maximum lightening areas from July in period-I to August in period-II. There is an increase in the intensities in the month of October in period-I also but it is not supported by LIS data which are less

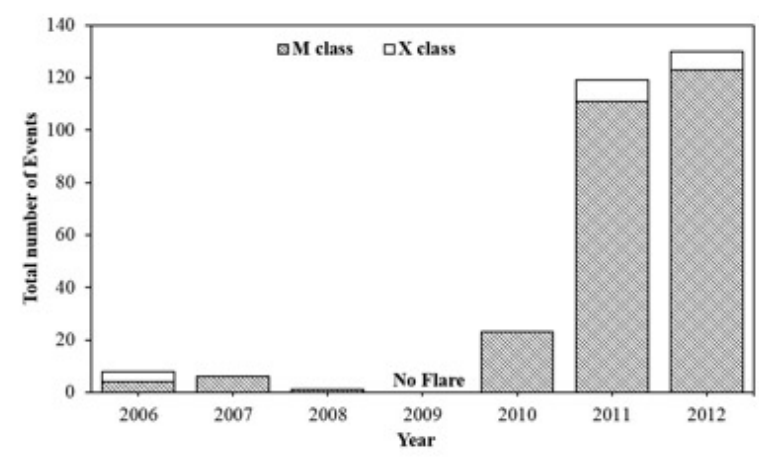

Figure 5. Variation of $\mathrm{M}$ and $\mathrm{X}$ classes of solar flares between the years 2006 and 2012.

than those in the month of July. The intensities fall in the southern hemisphere $(\mathrm{SH})$ summer (NovemberJanuary) because the thunderstorms move away from the observer as shown by the increasing trend in source-observer distance.

\section{Discussion}

As per NASA report the solar cycle minimum of 2008-2009 was extraordinarily deep and the sun was quietest ever seen in almost a century. The X-ray radiation, which ionizes the earth's atmosphere and is responsible for the conductivity profile of the ionosphere, was by an order of magnitude lower than the solar cycle minimum studied previously [Onraskova et al. 2011]. In Figure 5 we show the variation of total number of $\mathrm{M}$ and $\mathrm{X}$ classes of flares occurred between the years 2006 and 2012. These flares are shown by hatched and open portions of histograms respectively. As it may be seen from the figure, the number of the two classes of flares decreases towards the solar cycle minimum such that there is no flare of either class in 2009. Then the number increases as the solar cycle increases. The solar flare data are obtained from NOAA National Geophysical Data Center on the website: www.ngdc.noaa.gov. It is well known that according to classical Schumann formula, the eigen-frequencies are given by the expression $\mathrm{fn}=7.5 \sqrt{\mathrm{n}}$ $(n+1)$ which are $10.6,18.4,26 \ldots \mathrm{Hz}$ for a spherical Earth-ionosphere cavity. However, the real (experimental) values are $8,14,20 \ldots \mathrm{Hz}$. The deviations are caused by the influence of the finite conductivity of the ionosphere [Nickolaenko and Hayakawa 2002]. From Figure 1 it may be seen that peak frequencies of the different modes do not coincide with their corresponding customary frequencies but are lower. For example, the fundamental mode looks to be near $7.5 \mathrm{~Hz}$ (instead of closer to $8 \mathrm{~Hz}$ ), the third at $19 \mathrm{~Hz}$ rather than $20 \mathrm{~Hz}$, and the fourth at $25 \mathrm{~Hz}$ (instead of $26 \mathrm{~Hz}$ ). A possible reason for the low frequencies seems to be the fact that since the period of observation corresponds to the increasing phase of solar cycle minimum of 2008-2009, the effect of deep minimum activity still continues 
under which the modal frequencies are influenced. It may be mentioned here that Ondraskova et al [2011] have reported similar decrease in the frequencies of all the four modes from their observations at Modra observatory from solar cycle maximum of 2001-2002 to solar cycle minimum of 2008-2009. Satori et al [2005] have also reported a similar decrease in the frequencies. From Figure 4a we see that the peak frequencies of the first SR mode are much lower in period-II than those in period-I. The peak frequencies are lower than that of customary frequency of $8 \mathrm{~Hz}$ in period-I also. Reductions are also seen in peak frequencies of SR data obtained at Shillong station in India for the months of July and August 2007 and March and May, 2008 (Data obtained with the courtesy of Dr. B.M. Pathan, Indian Institute of Geomagnetism, Mumbai, May, 2010). Recently, Ondraskova et al. [2009, 2011] have reported a significant decrease of about $0.30 \mathrm{~Hz}$ in the fundamental SR frequency from observations at Modra observatory in Slovak Republic during the latest solar cycle having minimum around 2008-2009. This extraordinary fall of the fundamental mode frequency is attributed to the unprecedented drop in the ionizing radiation in X-ray frequency band, although the patterns of the daily and seasonal variation remain unchanged. Similar decreases in the first mode SR frequency have also been reported by Satori et al. [2005] for the previous 11 year solar cycle. Further decreases in frequency in period-II but having an increasing trend with the month may be interpreted in terms of the minimum solar cycle effect and increasing period of current solar cycle (2009-2020). According to Satori et al. [2005], changes of the X-ray radiation dominate the variations in the conductivity profile within the upper characteristic layer (90-100 Km portion of E-region). The decrease of this conductivity by up to one order of magnitude over the solar cycle is responsible for the observed SR frequency decrease by several tenths of $\mathrm{Hz}$. Although, there is a controversy that this is the intermediate region where electron conductivity is substituted by the ionic one. Therefore knee models of the conductivity profiles are used while modelling SR [e.g. Mustak and Williams 2002]. In general two possibilities have been suggested for the reduction in frequencies of the first mode; (i) variation in median distance between the source and the observer, and (ii) ionospheirc modification. Peak frequency changes to the extent of 10-20 percent have been observed which are attributed to the uncertainties arising from spatial distribution of lightning sources exciting the SR modes [De 2007]. De et al [2009] have also observed large diurnal variation in the peak frequency of the first mode and found similar variation in the data obtained at Moshiri in Japan. Model calculations have shown that a uniform decrease in the reference ionospheirc height reduces the resonant frequencies and $\mathrm{Q}$ factors (i.e. it simultaneously increases the wave attenuation). Specifically, it is found that a shift from 8 to $7.6 \mathrm{~Hz}$ in the peak frequency of the first mode is caused by a global reduction of ionospheirc height by approximately $5 \mathrm{Km}$. However, such reduction will be observed in all the modes, and the effect will be observed globally. Since measurements differ from place to place it is concluded that the deviations observed experimentally are conditioned by the displacement of the global thunderstorms rather than by modification of the global ionospheirc profiles [Nickolaenko and Hayakawa 2002]. The long-term observations of SR carried out by Ondraskova et al [2007] have also confirmed that variation in peak frequency of the lower SR modes can be attributed mainly to the source-observer distance effect. The intensity variation during the two periods (see Figure 2) is influenced by solar activities. Since the period I corresponds to close to solar cycle minimum of 2008-2009, the intensities are lower. In contrast, the period II corresponds to increasing solar cycle, the intensities are higher. These results are very well supported by the results of earlier workers. For example, Kulak et al. [2003] have analyzed six years of SR data at a mid-latitude station which included the periods of minimum to maximum (1995-2001) of solar cycle 23. The results of the analysis and modelling have shown that first SR frequency increases from $7.75 \mathrm{~Hz}$ at minimum to about $7.95 \mathrm{~Hz}$ at maximum while the global mean attenuation rate at $8 \mathrm{~Hz}$ varies from $0.31 \mathrm{~dB} / \mathrm{Mm}$ at minimum to about $0.26 \mathrm{~dB} / \mathrm{Mm}$ at maximum. Recently, Koloskov et al. [2013] have reported the results of long period (20022012) horizontal magnetic field SR observations conducted at Ukrainian Antarctic station "Academician Vernadsky" and shown that both the SR first mode frequency and horizontal component's intensity follow the trend in variation of solar activity. The LIS data relate to cloud formation and lightning flashes near the earth's atmosphere and are also influenced by solar activities. A detail discussion on this topic will follow later. A possible explanation for shifting of the peak thunderstorm activity from the month of July, 2007 in period-I to the month of August, 2011 in period-II may be given in terms of galactic cosmic ray (GCR) variation with solar activity. Many workers [Tinsley and Yu 2004, Siingh and Singh 2010, Siingh et al. 2011, Williams and Mareev 2014] have reviewed the cosmic ray- mediated cloud microphysics on the 11 year solar cycle. It has been mentioned that in all specific mechanisms, increased GCRs during solar minimum are linked with increased cloud condensation 
nuclei (CCN) and ultimately with increased cloudiness, and (in the atmospheric electrical context) with increased cloud water in the mixed phase region of moist convection [Williams et al. 2002]. This has been supported by other workers also [Pierce and Adams 2009, Erlykin and Wolfandale 2011].It is now a wellestablished fact that GCR flux is reduced as solar activity increases. This is because the increased solar magnetic flux associated with enhanced solar wind during high solar activity periods deflects the GCRs away from the heliosphere and hence from entering the earth's atmosphere. The reduced levels of GCRs modulated by increasing solar wind can significantly decrease cosmogenic ionization resulting in decreased conductivity and thus increase electric potential. Any significant change in electric field in the region of cloud formation can affect cloud formation, cloud movement etc. through a number of processes not yet understood [Lakshmi 2008]. The possible long term correlation between GCRs and low cloud cover (LCC) could be explained considering the influence of solar activity [Erlykin et al. 2009]. The enhanced solar irradiance causes a rise of the mean surface temperature and results in enhanced vertical convection. From below $3 \mathrm{Km}$ warm air rises to greater heights and causes the LCC to decrease and median cloud cover (MCC) to increase setting a change in vertical gradient of the electric field. Thus, during high solar activity periods the decrease in GCRs causes a decrease in LCC, although based on this argument no casual connection between GCRs and LCC could be established. Keeping the above arguments in view, it is possible that the intense level of lightning in the month of July in period-I is influenced by the increasing solar activity in period-II and shifted to the month of August in period-II. Since the increased solar activity during the period-II is responsible for decreased cloud formation, this would result in reduction in lightning activities and hence a reduction in LIS data as found in Figure 2 during period-II. However, this explanation is tentative and a detailed explanation for the fall of LIS data during period-II is under study. In all the results presented in Figure 4, we have adopted a single source model due to Nickolaenko and Hayakawa [2002] assuming that the global lightening can be approximated by a single source migrated in longitude around the earth. This is not a good model for the real global lightening source, which exists in three well recognized continental zones, none of which ever really goes to sleep over the course of 24 UT hours. Hence, a model incorporating the three sources is better and this has been recognized by Nickolaenko et al. [1998]. However, it is also far from the ideal because the source parameters depend (although slightly) on the particular field site. Applications of OTD/LIS data also does not provide one-to-one correspondence to observations. And also we do not know spatial distributions of strokes [A.P. Nickolaenko, personal communication, September 2014].

\section{Conclusion}

Employing a set of 3-component search coil magnetometer and analysing the X-component data, we study the variation of first mode SR intensity and frequency during two periods of 01 April, 2007 to 31 March, 2008 (Period I) and 01 March, 2011 to 29 February, 2012 (Period II) which correspond to pre and post periods of solar cycle minimum of 2008-2009. Using the intensity data, we first study the monthly distribution of global thunderstorm activity and then using both the frequency and intensity data we study the effective source distance and level of lightning during both the periods. We show that the world thunderstorm activity shifts to summer month in the northern hemisphere summer as the effective source distance approaches close to the observer, by 12 and $9 \mathrm{Mm}$ respectively in both the periods. The level of lightening in different months of the two periods are supported by the LIS data with correlation coefficients of 0.81 and 0.70 respectively. The level of intense lightning lies in the month of July, 2007 in period-I which shifts to August, 2011 in period-II. This shifting is attributed to increasing solar activity in period-II due to which cloud formation and movement are reduced and hence the effective lightning also is delayed.

Acknowledgements. The authors are highly grateful to the Department of Science and Technology, Government of India, New Delhi for the financial support in the form of major research project. The authors also thank Mr. Ajay Singh for typing the manuscript and help with the figures.

\section{References}

Balser, M. and C. A. Wagner (1960). Observation of Earth-ionosphere cavity resonances, Nature, 188, 638-641.

Bingham, C., M.D. Godfrey and J.W. Turkey (1967). Modern techniques of power spectrum estimation, IEEE trans. Audio and Electroacoust, AU-15, 56-66.

Christian, H.J., R.J. Blakeslee and S.J. Goodman (1989). The detection of lightening from geostationary orbit, J. Geophys. Res: Atmospheres (1984-2012), 94, 13329-13337.

Christian, H.J., R.J. Blakeslee, D.J. Boccippio, W.L. Boeck, D.E. Buechler, K.T. Driscoll, S.J Goodman, J.M. Hall, W.J., Koshak, D.M. Mach and M.F. Stewart (2003). Global frequency and distribution of light- 
ening as observed from space by the Optical Transient Detector, J. Geophys. Res., 108, doi: 10.1029/ 2002 JD002347.

De, S. S. (2007), Guest Editorial, Schumann resonance. Its different aspects and latest wonders, Indian J. Radio \& Space Phys., 36, 359-361.

De, S.S., B. K. De, B. K. Sarkar, B. Bandyopadhyay, D. K. Halder, P. Suman and S. Barui (2009), Analysis of Schumann resonance spectra from Kolkata and their possible inter pretations, Indian J. Radio \& Space Phys., 38, 208-214.

Erlykin, A.D., T. Sloan and A.W. Wolfendale (2009). The search of cosmic ray effects in the clouds, J. Atmos. Solar-Terr. Phys., 71, 955-958.

Erlykin, A.D. and A.W. Wolfendale (2011). Cosmic rays effects on the cloud cover and their relevance to cloud change, J. Atmos. Solar-Terr. Phys., 73 (13), 1681-1686.

Finke, U. and T. Hauf (2002). Detect and locate lightening events from geostationary satellite observations, report part II: Feasibility of lightening location from a geostationary orbit, No. EUM/CO/02/1016/SAT, Institute for Meteorologie und Krimatologie, University Hannover.

Greenberg, E. and C. Price (2007). Diurnal variation of ELF transients and background noise in the Schumann resonance band, Radio Sci., 42, RS2S08, doi: 10.1029/2006RS003477.

Hayakawa, M., K. Ohta, A. P. Nickolaenko and Y. Ando (2005). Anomalous effect in Schumann resonance phenomena observed in Japan, possibly associated with the Chi-Chi earthquake in Taiwan, Ann. Geophys, 23, 1335-1346.

Hayakawa, M., A.V. Shvets, Y. Hobara and A.P. Nickolaenko (2010). Recent studies of Schumann resonance and ELF transients, In lightening: properties, Formation and Types, chapter 3, Ed. (Mathew D. Wood), Nova Science Pub., London, pp: 1-33.

Koloskov, A.V., A.V. Spikelets, N.A. Baru, O. Budanov, V.G. Rutlers, B.J. Gavriliouk, A.V. Paznuhov and Y.M. Yampolsky (2013). Diagnosis of global lightening activity on the basis of long-term monitoring signals Schumann resonance on the UAS, Ukrainian Antarctic Journal, N.12, 170-176.

Kulak, A., J. Kubisz, A. Michalec, S. Zieba and Z. Nieckarcz (2003). Solar variation in extremely low frequency propagation parameters: 2 . Observation of Schumann resonances and compulation of the ELF attenuation parameters, J. Geophys. Res., 108, 1271, doi 10.1029/2002 J/A009305.

Lakshmi, D. R. (2008). A brief review of solar activity modulations of global electric circuit, in the proceedings of DST sponsored workshop on Electro- dynamical coupling of atmospheric regions, organized by Indian Institute of Geomagnetism, Navi Mumbai, 25-26, November, pp. 6-8.

Melnikov, A., C. Price and G. Satori (2004). Influence of solar terminator passages on Schumann resonance parameters, J. Atmos Solar-Terr. Phys., 66, 1187-1194.

Mustak, V.C. and E.R. Williams (2002). ELF propagation parameters for uniform models of the earthionosphere wave guides, J. Atmos. Solar-Terr. Phys., 64, 1989-2001.

Nickolaenko, A. P. and L. M. Rabinowicz (1995). Study of annual changes of global lightning distribution and frequency variations of the first Schumann resonance mode, J. Atmos. Solar-Terr. Phys., 57, 1345-1348.

Nickolaenko, A.P., G. Satori, V. Ziegler, L.M. Rabinowicz and L.G. Kudintseva (1998). Parameters of global thunderstorm activity deduced from the long-term Schumann resonance records, J. Atmos. Solar-Terr. Phys., 60, 387-399.

Nickolaenko, A. P. and M. Hayakawa (2002). Resonances in the earth-ionosphere cavity, Kluwer Academic Publishers, Dordrecht, the Netherlands.

Nickolaenko, A. P., M. Hayakawa, M. Sekiguchi, Y. Ando and K. Ohta (2006). Model modification in Schumann resonance intensity caused by a localized ionosphere disturbance over the earthquake epicenter, Ann. Geophys., 24, 567-575.

Nickolaenko A. P., O. Penchony and C. Price (2006), Model variations of Schumann resonance based on Optical Transient Detector maps of global lightning activity, J. Geophys. Res. 111, D23102, doi: 10.1029/ 2005JD006844.

Nickolaenko, A. P. and M. Hayakawa (2008). Universal and local time components in Schumann resonance intensity, Ann. Geophys., 26, 813-822.

Nickolaenko, A. P., E. I. Yatsevich, A. V. Shvets, M. Hayakawa and Y. Hobara, (2011). Universal and local time variations deduced from simultaneous Schumann resonance records at three widely separated observatories, Radio Sci., 46, 5, October, doi: 10.1029/2011RS004663.

Nickolaenko, A. P. (2014), Deducing the world thunderstorm activity from the Schumann resonance record (once again about point source model in Schumann studies), Radio Phys. and Electronics, 5(10), 33-41 (in Russian).

Ondraskova, A., P. Kostecky, S. Sevcik and L. Rosenberg (2007). Long term observation of Schumann resonance at Modra observatory, Radio Sc., 42, RS2509, doi: 10.1029/2006RS003478.

Ondraskova, A., S. Sevcik and P. Kostecky (2009). A significant decrease of the fundamental Schumann res- 
onance frequency during the solar cycle minimum of 2008-9 as observed at Modra Observatory, Contributions to Geophysics and Geodesy, 39/4, 345-354.

Ondraskova, A., S. Sevcik and P. Kostecky (2011). Decrease of Schumann resonance frequencies and changes in the effective lightening areas towards the solar cycle minimum of 2008-2009, J. Atmos. Solar Terr. Phys., 73, 534-543.

Pechony, O. and C. Price (2006). Schumann resonances: Interpretation of local diurnal intensity modulations, Radio Sci., 41, 2, doi: 10.1029/2006RS003455.

Pechony, O., Price C. and A. P. Nickolaenko (2007). Relative importance of the day-night asymmetry in Schumann resonance amplitude records, Radio Science, doi: 1029/2006RS003456.

Pierce, J.R. and P.S. Adams (2009). Can cosmic rays affect cloud condensation nuclei by altering new particle formation rates? Geophys. Res. Lett., 36 (LO 9820).

Price, C. and A. Melnikov (2004). Diurnal, seasonal and inter-annual variations of the Schumann resonance parameters. J. Atmos. Solar-Terr. Phys., 66, 1179-1185.

Satori, G. (2003). On the dynamics of the north-south seasonal migration of global lightning, Pro. of 12th ICAE, Versailles, France, global lightening and climate, pp. 1-4.

Satori, G., E. Williams and V. Mushtak (2005). Response of the Earth-ionosphere cavity resonator to the 11year solar cycle in X-radiation, J. Atmos. Solar-Terr. Phys., 67, 553-562.

Satori, G., M. Neska, E. Williams and J. Szendroi (2007a). Signatures of the day-night asymmetry of the Earthionosphere cavity in high time resolution Schumann resonance records, Radio Sci., 42, RS2510, doi: 10.1029/2006RS103483.

Satori, G., I. Lemperger and J. Bor (2007b). Modulation of annual and semiannual areal variations of global lightning on the 11-year solar cycle, In: Second International Symposium on lightening physics and effects, Vienna, April 19 and 20.

Satori, G., V. Moshtak and E.R. William (2009). Schumann resonance signatures of global lightning activity, In Lightning: Principles, instruments and applications [eds] Betz, H.D., Schumann, U., Laroche, P., Springer, pp. 641.

Schumann, W. O. (1952). On the free oscillations of a conducting sphere which is surrounded by an air layer and an ionosphere shell, Z. Naturforschaftung, 7a, 149-154.

Sentman, D. D. and B. J. Fraser (1991). Simultaneous observations of Schumann resonances in California and Australia: evidence for intensity modulation by the local height of the D region. J. Geophys. Res., 96 (A9), 15973-15984.
Siingh, D. and R.P. Singh (2010). The role of cosmic rays in the earth's atmospheric processes, Pramana, 74, 153-168.

Siingh, D., R.P. Singh, A.K. Singh, M.K. Kulkarni, A.S. Gautam and A.K. Singh (2011). Solar Activity, Lightening and climate, Article No. 912, Springer, doi: 10.1007/s 10712-011-9127-1.

Singh, B., R. Tyagi, Y. Hobara and M. Hayakawa (2014). $\mathrm{X}$-rays and solar proton event induced changes in the first mode Schumann resonance frequency observed at a low latitude station Agra, India, J. Atmos. SolarTerr. Phys., 113C, 1-9, doi: 10.1016/j.jstp.2014.02010.

Tinsley, B.A. and F. Yu (2004). Atmospheric ionization and clouds as link between solar activity and climate, In Solar variability and its effects on climate, Geophysical monograph No. 141, A.G.U., doi: 10.1029/141GM22.

Tyagi, R., B. Singh, O.P. Singh and R.L. Dua (2013). Characteristics of global thunderstorm extracted from Schumann resonance at Agra, Int. J. Adv. Res. in Electrical Electronics and Instrumentations Engg., 2(3), 1129-1137.

Welch, P. D. (1967). The use of Fast Faurier transform for the estimation of power spectra: A method passed on time averaging over short modified periodgrams, IEEE Trans. Audio and Electroacoust., AU-16, 70-73.

Williams, E.R. and Coauthors (2002). Contrasting connective regims over the Amazon: Implication to cloud electrification, J. Geophys. Res., 107, http://dx. doi.org/10.1029/2001JD000380 (LBA special issue, D20, 8081).

Williams, E.R. and G. Satori (2007). Solar radiation- induced changes in ionospheric height and the Schumann resonance waveguide on different timescales, Radio Sci., 42, RS2S11, doi: 1029/2006RS003494.

Williams, E. and E. Mareev (2014). Recent progress on the global electric circuit, Atmos. Res. (135-136), 208-227, doi.org/10.1016/j.armores. 2013.05.015.

\footnotetext{
${ }^{\star}$ Corresponding author: Birbal Singh, RBS Engineering Technical Campus, Department of Electronics and Communication Engineering, Bichpuri, Agra, India; email:bbsagra@gmail.com.

C 2014 by the Istituto Nazionale di Geofisica e Vulcanologia. All rights reserved.
} 